\title{
Diffusivity-dependent fracturing processes and microseismic activity in granite
}

\author{
Catarina Baptista-Pereira ${ }^{1 *}$ and Bruno Gonçalves da Silva ${ }^{1}$ \\ ${ }^{1}$ New Jersey Institute of Technology, Newark, New Jersey, United States of America
}

\begin{abstract}
Enhanced Geothermal Systems have relied on hydraulic fracturing to increase the permeability of rock reservoirs. The permeability enhancement depends on the connectivity between new and existing fractures. This, in turn, depends to a large extent on the interaction between the rock and the fracturing fluid, which not only pressurizes existing and new fractures but also diffuses into the rock matrix. In this research, the effect of the diffusivity of hydraulic oil on the fracturing processes and microseismicity of unconfined prismatic granite specimens was experimentally evaluated using visual and acoustic emission monitoring. The tests consisted of injecting hydraulic oil into two pre-fabricated flaws at two rates $(2 \mathrm{ml} / \mathrm{min}$ and 20 $\mathrm{ml} / \mathrm{min}$ ), kept constant in each test. The fluid pressure inside the flaws was increased until hydraulic fractures propagated and the fluid front growing from the pre-fabricated flaws was visually monitored throughout the tests. It was observed that the fracturing pressures and patterns were injection-rate-dependent, which shows that diffusivity and poro-elastic effects play an important role in the hydraulic fracturing processes of granite. A smaller fluid front was observed for the $20 \mathrm{ml} / \mathrm{min}$ injection rate, associated to a lower volume injected and to a higher fracturing pressure when compared to the $2 \mathrm{ml} / \mathrm{min}$ injection rate. This was interpreted to be caused by the different pore pressures that developed inside of the rock matrix, which are function of the fluid front size. Microseismic activity was observed throughout the tests, becoming more intense and localized near the flaws as one approached the end of the test (i.e. visible crack propagation). While microseismic events were observed outside the fluid front region, their density was significantly larger within this area, showing that fluid diffusivity may contribute to an intensification of the microseismic activity.
\end{abstract}

\section{Introduction}

Hydraulic fracturing is a technique used since the 1940's to increase oil and gas production [1] and has also become a topic of significant interest for enhance geothermal recovery [2], due to the increase of energy demand in the US and worldwide. According to Strain [3], the hydraulic fracturing method was formalized in 1957-1958, and consists of injecting a fluid at a certain rate and pressure into a borehole or well, to enable the opening of new fractures and mobilization of existing ones [4,5]. This results in the increase of the permeability of the rock or reservoir matrix, as well as in localized increases of pore pressures. Simultaneously, fluid diffusing into the rock matrix may lead to fluid losses and affect the breakdown pressure and fracturing processes [6]. Moreover, hydraulic fracturing is commonly associated to microseismic activity which occurs when fractures are created or reactivated. This paper aims to evaluate the effect of fluid diffusivity into the rock matrix on the fracturing processes and microseismicity.

\subsection{Fluid-induced microseismicity}

Whenever a rock is stimulated to create and/or reactivate in-situ fractures and therefore to increase its permeability, there is an increase in microseismic events [7]. In fact, this microseismicity activity has been used in recent years to evaluate the complexity of the rock fractures, including the interaction between hydraulic and existing fractures [8]. It is also known that the increase in pore pressures may result in the fracturing of porous rocks and in the consequent increase in microseismic activity [9]. Therefore, it is important to evaluate the relationship between the fluid-induced seismic events and fracturing processes [7], as will be discussed in this paper.

\subsection{Fluid diffusion}

As previously mentioned, hydraulic fracturing consists of injecting fluid into a reservoir rock, which may cause some of the fluid to penetrate the interconnected pores of the rock matrix [4][10]. This will result in an increase in pore pressures, and consequent decrease of effective stresses; however, it is not fully understood how fluid diffusion changes the fracturing behavior when hydraulic fracturing is employed.

Haimson and Fairhurst [11] developed relationships between in-situ stresses and breakdown pressures taking into account fluid diffusivity into the rock matrix for constant injection rates. Carcione et al. [12] numerically showed that changes in the effective stresses induce microseismic events in a rock reservoir, and that the permeability and hydraulic diffusion depend on these

\footnotetext{
* Corresponding author: cb373@njit.edu
} 
pressures. Bruno and Nakagawa [5] state that hydraulic fractures are influenced by pore pressures around the fracture tips, as well as by the orientation and distribution of pore pressure gradients. These authors investigated how non-uniform pore pressure fields influence the direction of fracture propagation, concluding that the strain energy release rate is maximum in areas with high local pore pressures, which leads to the development of tensile cracks. By studying the influence of injecting nitrogen on sandstone specimens, Hou et al. [4] observed that the breakdown pressure decreases with decreasing gas diffusion and that the fracture patterns are influenced by pore pressures. Indeed, not only did they observe that pore pressures have a more significant impact for low injection rates, they also found that high pore pressures promote micro-crack initiation and are associated with a more complex network of fractures.

\subsection{Current paper}

The current study builds upon the experimental work developed by Baptista-Pereira and Gonçalves da Silva [13], but using granite instead of gypsum specimens. The present paper investigates the effect of fluid diffusivity on the hydraulic fracturing mechanisms, by pressurizing a couple of pre-fabricated flaws using two different constant injection rates of 20 and $2 \mathrm{ml} / \mathrm{min}$. Fracturing mechanisms were investigated by relating fluid pressure variation with visual and acoustic emission data. The paper is organized as follows: in Section 2, the methodology is presented, which includes the specimen preparation, the description of the pressure enclosure and the test setup used in the experiment, as well as the procedures employed to interpret the tests. Section 3 discusses the fracturing pressures, fluid fronts and fracturing patterns, as well as the microseismic activity. Finally, Section 4 presents the summary and conclusions.

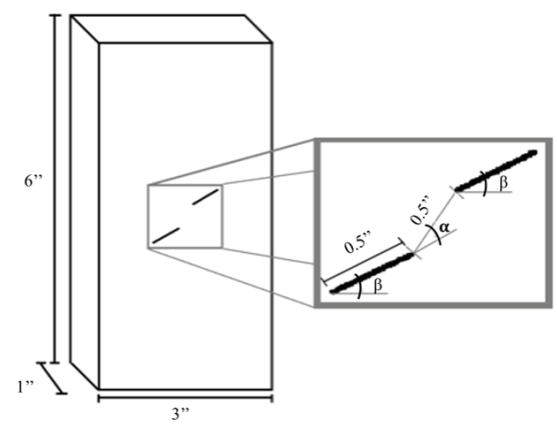

Figure 1: Dimensions of specimen and its pre-fabricated flaws ( $\beta=\alpha=30^{\circ}$ and length of $\left.0.5^{\prime \prime}\right)$ [13]

\section{Methodology}

\subsection{Specimen preparation}

The tests were conducted on granite specimens with the dimensions shown in Figure 1. A waterjet was used to cut the pre-fabricated flaws, since this cutting method prevents significant damage around the flaw tips. The properties of the Barre granite specimen are described in detail in [14], based on several studies conducted by the MIT rock mechanics group and by the geo-resources and geotechnical laboratory at NJIT.

\subsection{Test setup}

The specimen was placed inside a pressure enclosure described by Gunarathna and Gonçalves da Silva [15], and is shown in Figure 2. The enclosure is a three-plate chamber, with two outer O-rings (one in the front and one in the back faces of the specimen), which seal the specimen. Hydraulic oil was injected into the enclosure and the flaws were pressurized at a constant rate of either 2 or $20 \mathrm{ml} / \mathrm{min}$ by using a Teledyne $\mathrm{ISCO}^{\mathrm{TM}}$ syringe pump that was connected to the back-steel plate of the enclosure. This syringe pump allowed one to apply a fluid pressure to the flaws, which was measured by a pressure transducer and was logged by a data acquisition system that recorded these data during the experiment (Figure 4).

The fluid front that grew from the flaws into the rock due to the diffusivity of the hydraulic oil was visually monitored thanks to an impermeable transparent membrane attached to the front and back of the specimen, as shown in Figure 2. By applying this membrane, the fluid pressure was applied in the internal surfaces of the flaws and in the back and front surfaces of the specimen; however, the fluid diffused only through the inner surfaces of the pre-fabricated flaws, as shown in Figure 5.

\subsubsection{Visual and microseismic monitoring}

During the test, a high-speed and a high-resolution cameras monitored the damage initiation and propagation during the entire experiment and in the last $2.5 \mathrm{sec}$ of the test, respectively. Eight wideband (optimum response from $100 \mathrm{kHz}$ to $900 \mathrm{kHz}$ ) acoustic emission sensors (two in each lateral surface of the specimen, as shown in Figure 3) were used to monitor the microseismic activity generated throughout the test. This equipment is fully described by Gunarathna and Gonçalves da Silva [15].

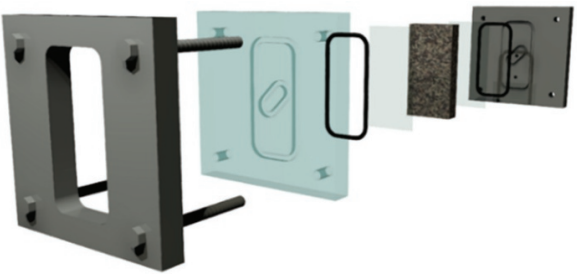

Figure 2: Pressure enclosure setup

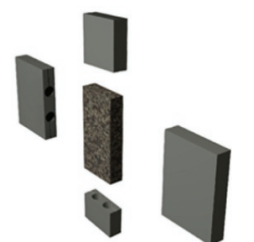

Figure 3: Location of the AE sensors

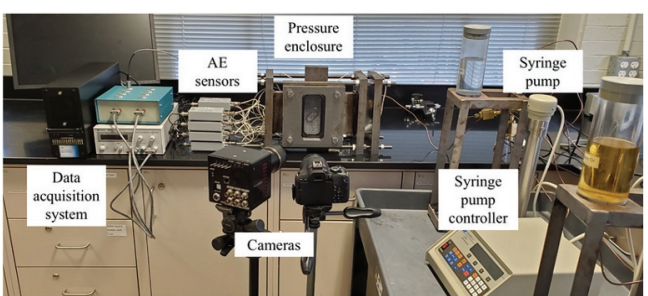

Figure 4: Enclosure and setup used in the experiments 


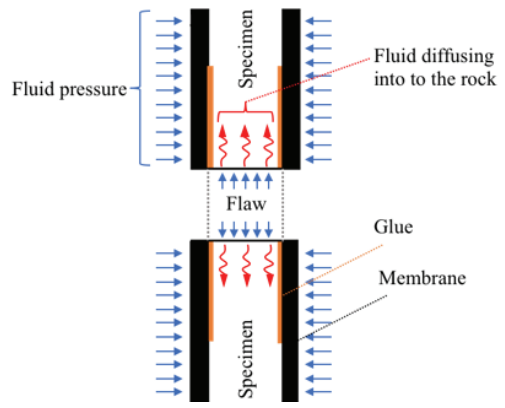

Figure 5: Cross-section of the specimen showing the membrane used to seal the faces of the specimen, based on [13]

\subsection{Analysis of the experiments}

In order to understand the initiation, propagation and coalescence of cracks due to the increase of both pressure inside the flaws and pore pressures in the rock matrix, a visual analysis was conducted by analyzing pictures taken throughout the experiment. These images allowed one to observe the fluid growth and relate it to the variations in the fluid pressures.

Location, rate and first P-wave amplitudes of the microseismic events were also related to the injected volume, pressure, fluid fronts and visible damage, observed throughout the tests.

\section{Experimental results}

To easily identify the four tests, the specimens follow the notation shown in Table 1. Sections 3.1, 3.2 and 3.3, will describe the pressure variation, visual observations and microseismic activity, respectively.

Table 1: Identification of the granite specimens tested

\begin{tabular}{|c|c|c|}
\hline Rate $(\mathrm{ml} / \mathrm{min})$ & Specimen 1 & Specimen 2 \\
\hline 20 & GR $20-1$ & GR $20-2$ \\
\hline 2 & GR $2-1$ & GR $2-2$ \\
\hline
\end{tabular}

\subsection{Pressure variation of injected fluid}

Figures 6 and 7 show the variation of pressure with time and volume, respectively, for the injection rates of 20 and $2 \mathrm{ml} / \mathrm{min}$. In Figure 6, similar to what was observed in

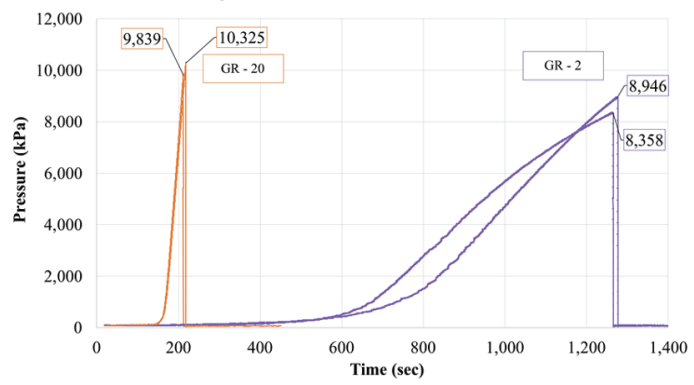

Figure 6: hydraulic oil pressure versus time for the four specimens tested using two different injection rates

\subsection{Visual observations}

This subsection evaluates the fluid fronts and fracturing patterns that are observed when the maximum fluid gypsum by Baptista-Pereira and Gonçalves da Silva [13], the experimental data suggests that three major stages can be identified:

- 1st stage: pressures are approximately zero, corresponding to the filling of enclosure;

- 2nd stage: pressure increase, allowing injection fluid to start diffusing into the rock matrix;

- 3rd stage: fracturing of the specimen, after the maximum pressure is reached, followed by a quasiinstantaneous pressure loss.

For the high injection rate, visible cracks develop earlier than for the low injection rate, as intuitively expected. Indeed, fracturing occurs at an average time of 215.21 and $1,270.12 \mathrm{sec}$, for the high and low injection rates, respectively.

The average fracturing pressures for high and low injection rates are $10,082 \mathrm{kPa}$ and $8,652 \mathrm{kPa}$, respectively. Baptista-Pereira and Gonçalves da Silva [13] observed that the fracturing pressures in gypsum are affected by the fluid front that diffused through the rock matrix in the bridge between the inner tips of both flaws. This is also observed in the current granite experiments, as will be further discussed in Section 3.2.

The following can be observed in Figures 6 and 7: i) for high injection rates (GR-20), both tests are very similar, with the pressure increasing with time and volume injected in an almost linear manner once the enclosure is filled. In the early stage of the experiment, a positive convexity is observed, corresponding to the filling of enclosure. After this period the pressure increased linearly until it eventually drops to zero almost instantaneously; ii) for low injection rates (GR-2), the initial "enclosure filling" stage takes longer to develop, as expected. In the second stage, the linear behavior is followed by a non-linear segment with a negative convexity. This may be due to i) progressive microdamage of the granite, which may have more time to develop when lower injection rates are used and/or ii) a larger volume of fluid being lost into the specimen by diffusivity. In fact, the average injected volume is $31 \mathrm{ml}$ for the lower injection rate, which represents an increase of $35 \%$ when compared to high injection rates.

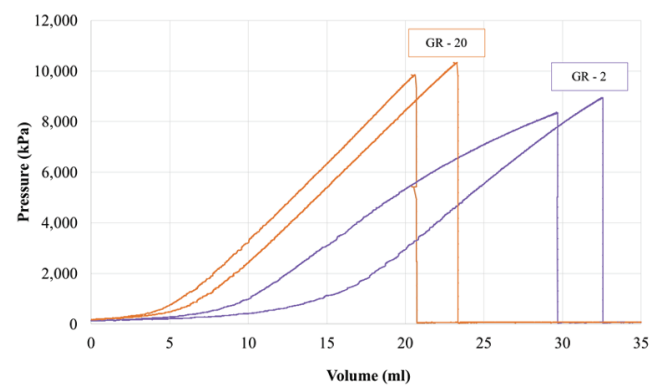

Figure 7: hydraulic oil pressure versus volume injected for the four specimens tested using two different injection rates

pressure is reached (i.e. fracturing pressure). Figures 8 and 9 represent the fluid fronts at failure and fracturing patterns for the injection rates of 20 and $2 \mathrm{ml} / \mathrm{min}$, respectively. 


\subsubsection{Fluid fronts}

Figures 8 and 9 show that the fluid fronts are larger for the lower injection rates. Moreover, instead of a uniform elliptical fluid front growth, as observed in gypsum [13], the fluid growth is very irregular in the granite specimens tested. The morphology of these rocks may explain the observed differences in fluid front growth. Indeed, the main differences between the gypsum and granite are their grain size, mineral composition and porosity. Gypsum represents a more porous and homogeneous material and granite is less permeable and more heterogeneous,

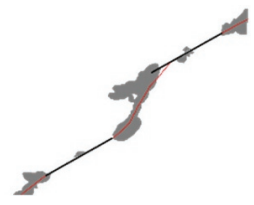

a) GR20 - 1

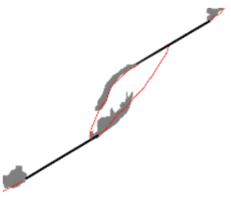

b) GR20-2
Figure 8: Fluid fronts (dark grey) and fracturing patterns (red), when oil is injected at a constant rate of $20 \mathrm{ml} / \mathrm{min}$

\subsection{Microseismic activity}

The following section evaluates the time-variation and the effect of the injection rate on the microseismic activity observed in the tests.

\subsubsection{Time variation}

Four main stages are identified for both injection rates, as shown in Figures 10 and 11, which have two vertical axes, corresponding to the number of $\mathrm{AE}$ events and to the first P-wave amplitudes.

- $\quad$ Stage 1: from the start of the test to when the AE events start to occur at a higher rate, corresponding to the beginning of the tests. The average number of events recorded is relatively small.

- $\quad$ Stage 2: from when the AE events start to occur at a higher rate to the development of the visible fractures. The end of this stage is marked by reaching the maximum rate of $\mathrm{AE}$ events and the maximum fluid pressure.

- $\quad$ Stage 3: from the time when the fracturing pressure is reached to when the rate of $\mathrm{AE}$ events decreases to almost zero. During this stage, the number of AE events decreases substantially.

- $\quad$ Stage 4: from the time when the rate of AE events decreases to approximately zero to the end of the test.

The major similarities and contrasts between the microseismic activity for the two injection rates tested will now be discussed for the identified stages. The AE amplitudes were evaluated according to the maximum value reached in each stage defined in Figures 10 and 11. - Stage 1: For both injection rates, the behavior is similar. However, it is noted that this stage is much longer for the lower than for the higher injection rate, corresponding to $86 \%$ and $58 \%$ of the total experiment duration, respectively. For high injection rates, there is no evidence of fluid fronts at this stage, but for the lower injection rate, there is already evidence of fluid diffusing into the rock matrix. For the higher injection rate, stage 1 composed of different mineral grains that also differ in size, which may lead to a more irregular fluid growth.

\subsubsection{Damage and fracturing}

In terms of fracturing patterns, it is noted that the fracturing coalescence is not as consistent as for gypsum [13] for both injection rates. When comparing Figures 8 and 9 , it is visible that the coalescence occurs through either one or two visible cracks, independently of the injection rates applied. This observation appears to indicate that fluid diffusivity may have a less significant effect on fracturing patterns in granite when compared to gypsum.

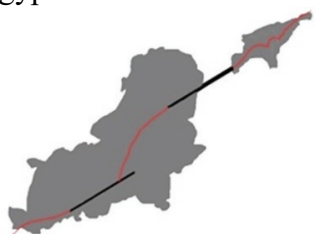

a) GR2 - 1

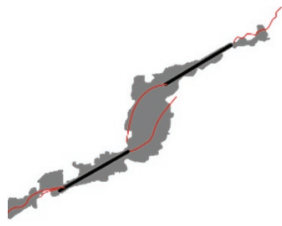

b) GR2-2
Figure 9: Fluid fronts (dark grey) and fracturing patterns (red), when oil is injected at a constant rate of $2 \mathrm{ml} / \mathrm{min}$

showed the lowest AE amplitudes (maximum of 108 and $92 \mathrm{~dB}$ for GR20-1 and GR20-2, respectively), while it showed the largest amplitudes for the lower injection rate (maximum of 131 and $117 \mathrm{~dB}$ for GR2-1 and GR2-2, respectively).

- $\quad$ Stage 2: For both injection rates, most AE events occur during this stage, with an average of 963 and 1,293 events for 20 and $2 \mathrm{ml} / \mathrm{min}$, respectively. When comparing the duration of this time window, it can be observed that it represents an average of $28 \%$ of the total experiment for the higher injection rate, while only $4 \%$ for the lower injection rate. For the higher injection rate, the fluid starts to diffuse into the matrix at 194 and 210 sec, for GR20-1 and GR20-2, respectively, while the fluid starts to be visible at Stage 1 for the lower injection rate. Regarding the $\mathrm{AE}$ amplitudes, their maximum values are $136 \mathrm{~dB}$ (GR20-1) and $121 \mathrm{~dB}$ (GR20-2) for the higher injection rate, which correspond to the highest values reached during the entire experiment. These large amplitudes appear to occur when the fluid front starts to be visually observed, which shows that there is a relation between the fluid diffusivity and magnitude of microseismic activity. For low injection rates, on the other hand, the $\mathrm{AE}$ amplitudes correspond to an average maximum value of $115 \mathrm{~dB}$, smaller than the average observed in Stage 1.

- $\quad$ Stage 3: For both injection rates, AE events are still recorded after fracturing and coalescence occurs, even though less frequently. In this stage, the $\mathrm{AE}$ amplitudes reach an average maximum value close to $120 \mathrm{~dB}$, which makes it the second stage with the highest AE amplitudes recorded.

\subsubsection{Location of events}

In order to analyze the relation between the fluid fronts and the AE events, the microseismic activity is assessed in Figures 12 and 13, where the AE events are shown for the full tests. These figures show that most AE events are 
localized next to the pre-fabricated flaws and near the hydraulically-induced fractures. It is observed that higher injection rates are associated with less $\mathrm{AE}$ events and with smaller fluid fronts. Indeed, the number of AE events are concentrated near the visible cracks, which developed in the bridge area and from the outer tips of the two flaws. It is also noted that, even though many $\mathrm{AE}$ events are
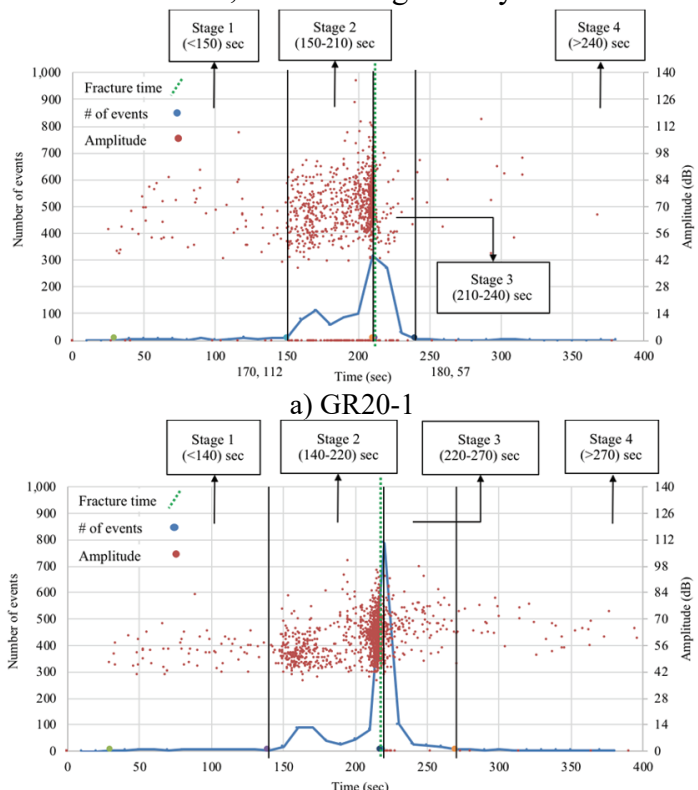

b) GR20-2

Figure 10: First $\mathrm{P}$-wave amplitude vs time, when oil is injected at a constant injection rate of $20 \mathrm{ml} / \mathrm{min}$
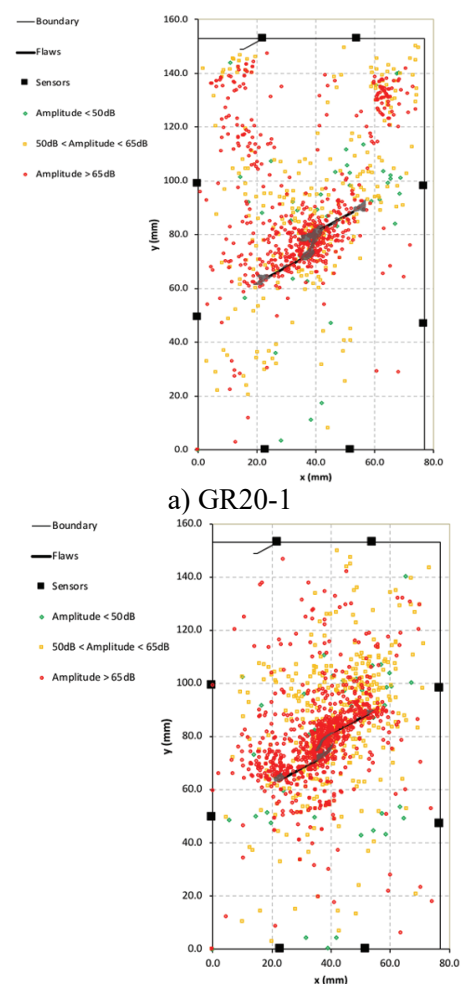

b) GR20-2

Figure 12: Microseismic activity, when oil is injected at a constant injection rate of $20 \mathrm{ml} / \mathrm{min}$ localized outside the fluid front region, they are significantly denser within this region. In fact, this dense microseismic region seems to be larger for wider fluid fronts. These two observations suggest that fluid diffusivity and associated poro-elastic effects may contribute to an intensification of microseismic activity.
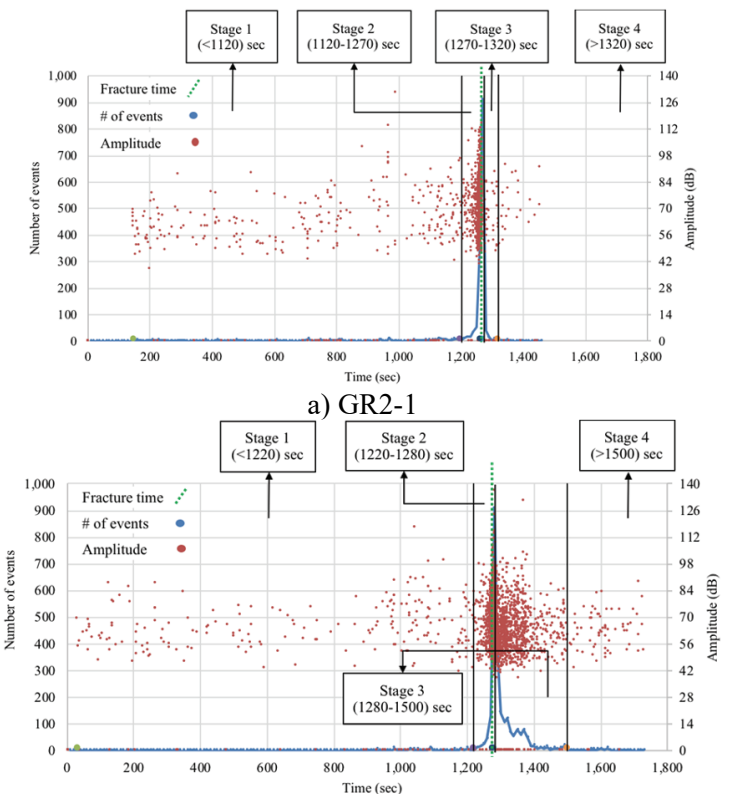

b) GR2-2

Figure 11: First P-wave amplitude vs time, when oil is injected at a constant injection rate of $2 \mathrm{ml} / \mathrm{min}$
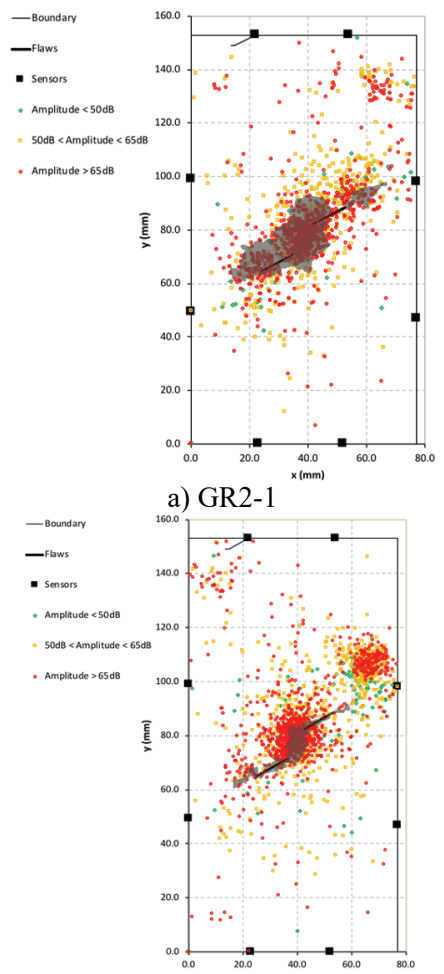

b) GR2-2

Figure 13: Microseismic activity, when oil is injected at a constant injection rate of $20 \mathrm{ml} / \mathrm{min}$ 


\section{Summary and conclusions}

This research investigated the diffusivity-dependent fracturing processes and microseismic activity in granite, while hydraulic oil was injected into two pre-fabricated flaws at two different injection rates of either 20 or 2 $\mathrm{ml} / \mathrm{min}$. Using an impermeable transparent membrane sealing the front and back faces of the specimen allowed to visually observe the fluid growth as it diffuses into the rock matrix. By using $\mathrm{AE}$ sensors, it was possible to monitor and to record the events that occurred throughout the experiments.

\subsection{Visual observations}

The fracturing pressures were larger and the injected volume was smaller for the higher injection rate, similar to what was observed in gypsum [13].

The fluid fronts for both injection rates showed an irregular growth during the entire experiment, which appeared to be related to the heterogeneity of granite, which was composed of different minerals with varying sizes. On the other hand, the higher injection rate corresponded to a smaller fluid front, since the fluid has limited time to diffuse into the rock matrix. The fluid diffusivity appeared to have little effect on the coalescence patterns for granite, as opposed to what was recently observed for gypsum.

\subsection{Microseismic activity}

Most AE events occurred until the end of Stage 2 or beginning of Stage 3, which was the time interval when there was a substantial growth of events and when the visual fracturing initiated and propagated. This stage was also characterized by a relatively large fluid front, indicating a relation between the microseismic activity and the poro-elastic effects caused by the diffusivity of the fluid into the rock.

For a low injection rate, the maximum AE amplitude was achieved during Stage 1. On the other hand, the diffusion of the fluid only occurred during the Stage 2 for a higher injection rate, which was when the maximum $\mathrm{AE}$ amplitude was reached. Once again, this showed a relation between a stronger microseismic activity and the fluid diffusivity.

For a low injection rate, Stage 2 was much shorter, comparatively to the higher injection rate (average of 28 $\%$ for $20 \mathrm{ml} / \mathrm{min}$ and of $4 \%$ for $2 \mathrm{ml} / \mathrm{min}$ ), which can be explained by the larger fluid front areas, which corresponded to more injected volume.

Stage 3, which initiated right after visible fracturing and pressure loss, was marked by a rapid decrease in the number of AE events. During this stage, the maximum AE amplitudes for both injection rates were relatively similar. Finally, most events were recorded next to the inner tips of both pre-fabricated flaws and in the bridge between them, which typically corresponded to the location where visible fracturing occurred. It was also noted that, even though many AE events were localized outside the fluid front region, they were significantly denser within this region. This suggests that fluid diffusivity may have contributed to an intensification of microseismic activity.

\section{References}

[1] B. C. Haimson, The hydraulic fracturing method of stress measurement: theory and practice. Pergamon Press Ltd, 1993.

[2] G. Zhai, M. Shirzaei, M. Manga, and X. Chen, "Pore-pressure diffusion, enhanced by poroelastic stresses, controls induced seismicity in Oklahoma," vol. 116, no. 33, 2019.

[3] H. J. Strain, "Well-bore notching and hydraulic fracturing," J. Can. Pet. Technol., vol. 1, no. 4, 1962.

[4] P. Hou, F. Gao, Y. Gao, Y. Yang, and C. Cai, "Effect of pore pressure distribution on fracture behavior of sandstone in nitrogen fracturing," Energy Explor. Exploit., vol. 35, no. 5, pp. 609626, 2017.

[5] M. S. Bruno and F. M. Nakagawa, "Pore pressure influence on tensile fracture propagation in sedimentary rock," Int. J. Rock Mech. Min. Sci., vol. 28, no. 4, pp. 261-273, 1991.

[6] S. A. Shapiro, C. Dinske, and E. Rothert, "Hydraulic-fracturing controlled dynamics of microseismic clouds," vol. 33, no. April, pp. 1-5, 2006.

[7] H. Barthwal and M. van der Baan, "Role of fracture opening in triggering microseismicity observed during hydraulic fracturing," 2019.

[8] S. C. Maxwell, T. I. Urbancic, N. Steinsberger, and R. Zinno, "Microseismic Imaging of Hydraulic Fracture Complexity in the Barnett Shale," Proc. - SPE Annu. Tech. Conf. Exhib., pp. 965-973, 2002.

[9] S. Turuntaev, O. Melchaeva, and E. Zenchenko, "Formation Fracturing by Pore Pressure Drop (Laboratory Study)," Intech, vol. 51, pp. 9931011, 2013.

[10] T. Ito, "Effect of pore pressure gradient on fracture initiation in fluid saturated porous media: Rock," Eng. Fract. Mech., vol. 75, no. 7, pp. 1753-1762, 2008.

[11] B. Haimson and C. Fairhurst, "Hydraulic Fracturing in Porous-Permeable Materials," $J$. Pet. Technol., vol. 21, no. 07, pp. 811-817, 1969.

[12] J. M. Carcione, G. Currenti, L. Johann, and S. Shapiro, "Modeling fluid injection induced microseismicity in shales," J. Geophys. Eng., vol. 15, no. 1, pp. 234-248, 2018.

[13] C. Baptista-Pereira and B. Gonçalves da Silva, "Effects of fluid diffusivity on hydraulic fracturing processes using visual analysis," $53 \mathrm{rd}$ U.S. Rock Mech. Symp., 2019.

[14] J.T. Miller, "Crack Coalescence in Granite B.S. Thesis," Massachusetts Institute of Technology, 2008.

[15] G. S. Gunarathna and B. Gonçalves da Silva, "Effect of the state of stress in the fracturing and micro-seismic activity of hydraulically-fractured granite," 53rd U.S. Rock Mech. Symp., no. September, 2019. 